\title{
WITH EQUALIZED MASS, ITS DENSITY OF MATTER CAN AFFECT RADIAL GRAVITATIONAL INTERACTIONS TOO
}

\author{
Jakub Czajko \\ Science/Mathematics Education Department \\ Southern University and A\&M College, Baton Rouge, LA 70813, USA \\ E-mail address: sunswing77@netscape.net
}

Keywords: Equalized mass; hidden variable; angular nonradial potential; pull-and-twist gravity.

\begin{abstract}
It is shown that if all stars and planets were made out of the same material substance, such as water, for example, then their density of matter, which is conventionally defined as the ratio of their actual mass to an equivalent mass of water, must be taken into account also in the usual radial Newtonian law of gravitation. The idea of equalized mass supports the concept of quantity of matter involving density of matter as well as the bulk/mass that Newton envisaged. It has been proved necessary to explain formerly unexplained experimental results, including discrepancies between predictions based on general theory of relativity (GTR) and actually recorded data. The equalized mass is not meant to turn us back and embrace post-Newtonian theories of gravitation, but to complement the (radial-only by design) GTR for the phenomena that are only partly radial and partly nonradial. A certain new pull-and-twist equation of orbital gravitational interactions is proposed.
\end{abstract}

\section{INTRODUCTION}

In the very first definition of his Principia Newton has ingeniously defined the concept of quantity of matter as determined jointly by density of the material substance that things are made of and by its bulk, which is known as mass [1]. Yet in primary equations of all gravitational theories developed before $2000 \mathrm{AD}$ - when I have demonstrated that various experimental evidence clearly suggested that density of matter of the mass that creates the radial/center-bound gravitational force field affects nonradial effects happening in the field [2] - density of matter was not included.

Newton has postponed publication of his Principia by some 20 years, presumably dissatisfied with his inability to answer at least two crucial questions. Firstly, why universe has not collapsed if - as his theory claimed - gravitational force fields are always attractive; and secondly: why density of matter seemingly did not really matter, for the equation of motion derived from his gravitational theory made quite correct prediction of the motion of moon and other planets without accounting for the density of matter.

Despite mounting experimental evidence that Newton was right [2], [3], mainstream scientists ignored it and left some childishly simple experiments unexplained and unreconciled [2]. Instead of taking seriously the very first definition that Newton has offered in his Principia, physicists and mathematicians "rationalized" his insight and in doing so created few nonsenses and even fake theorem [4].

Nevertheless, presence of nonradial effects of radial force fields resolved the two issues that bothered all pre-2000 AD theories of gravitational interactions. The new nonradial effects showed that density of matter does matter indeed for nonradial gravitational interactions and that they give rise to clearly repulsive nonradial gravitational interactions as well [5], [2], [3], [6], [7].

Let us introduce the concept of equalized mass, by which I mean a theoretical construct: It is a certain effective mass that could be assigned each celestial body if it would be composed of the same kind of material substance. For the solar system, for instance, equalized mass could be standardized if all planets and our Sun would be made of the same substance (let's say water in normal circumstances, since conditions are different). In other words, equalized mass presumes the 
same constitution of all material bodies within the given system. On molecular and atomic scales, however, the internal structure of atoms and molecules should also be taken into account.

At a deeper level of inquiry, of course, even for the solar system the internal structures of planets as well as the shapes of their fields (and surroundings - such as their satellites and rings) should be taken into account too. For relativistic investigations, also the parameters of motion (i.e. velocities and accelerations) relative to the center of the given system should also be taken into account. Notice that any meaningful considerations of shapes presumes taking care of structures and distributions of their density of matter or charge. Former physics already considered shapes at quantum level, but there was no known dependency of interactions on density of matter in the classical equations that quantum mechanics inherited from classical physics.

In this note I will show that if mass would be equalized so that all celestial objects could be considered as if made of the same material substance (and consequently having the same average density of matter) then the density of matter would have to be included into the usual radial gravitational force field as well. In other words: Density of matter has already been as if implicitly included in the old Newtonian equation of gravitational force by neglecting explicit recognition of the matter density's impact on nonradial gravitational interactions. Hence the density of matter was virtually present as a hidden variable in the Newtonian gravitational equation, but its presence was inadvertently concealed in the traditional formulation of the - radial by default - Newtonian gravitational force field. This assertion agrees with the mathematical discovery of density of matter as a hidden variable of macrophysics, or more precisely as hidden function-variable [8].

Since the necessity to take density of matter into account has been confirmed by experiments [2], [3] as well as by purely mathematical analyses of gravitational interactions [9], [8], it may be beneficial to briefly highlight some problems created by ignoring the Newton's ingenious definition and then concisely recapitulate the developments that actually resolved those problems.

\section{RECAPITULATION OF MISDEFINED POTENTIAL ENERGY}

Even nonmathematician can see that since the operationally correct (and mathematically legitimate) product differentiation rule (PDR) that is written here in terms relevant to radial force fields of a vector of the gravitational force $\mathbf{F}$ and the conventional distance pointing vector $\mathbf{r}$ as:

$$
\mathrm{dW}(\mathbf{F} \cdot \mathbf{r}):=\mathrm{d}(\mathbf{F} \bullet \mathbf{r})=\mathbf{F} \cdot \mathrm{d} \mathbf{r}+\mathbf{r} \cdot \mathrm{d} \mathbf{F}
$$

which is proven true (and thus is absolutely mandatory to be always applied for any products), then the traditional definition of work done rate (meant as the scalar product of a force vector $\mathbf{F}$ over the straightlinear distance $\mathbf{r}=|\mathbf{r}|$ to the given trajectory/orbit pointed by the distance pointing vector $\mathbf{r}$, yet mistakenly designated as the product of force over distance change rate dr instead):

$$
\mathrm{dW}:=\mathbf{F} \cdot \mathrm{d} \mathbf{r}
$$

is incomplete, and so is also the potential energy that is spent on the work done by the given force field. Yet the operationally wrong and conceptually faulty formula (2) is used in all textbooks and lectures [4] despite the fact that - according to ingenious Frenet-Serret (FS) formulas of differential geometry - the formerly omitted nonradial term $\mathbf{r} \bullet \mathbf{d F}$ is unavoidable in general [9].

How could that happen? Presumably because the FS formulas arrived too late (around 1848 AD), whereas Lagrange has already in $1773 \mathrm{AD}$ discovered that the function (2), which represents only radial part of total potential (energy) though, solves Laplace equation [10]. Lagrange realized that gravitational force field is a geometric construct, but his great achievement has been (probably inadvertently) misrepresented by interpreting the radial-only generic scalar potential $\mathrm{V}(\mathrm{r})=1 / \mathrm{r}$ as if it was everything there is to the concept of potential. Even visual comparison of the equations (1) and (2) indicates that one must not make such generalizations. Besides, every particular solution of a given differential equation contributes to its more general or prospective total solution. Hence no 
matter how one approaches the issue, the definition (2) either blatantly violates or discredits proven mathematical rules.

From a mathematical point of view, mass and scalar potential are not always scalars but could be considered as vectors in the direction of time [11], [12] - compare also [13]. In spacetime with $n$ time coordinates inertial and gravitational masses can be represented as $n \times n$ matrices [14]. These declarations are not just empty speculations. It has been shown that nonabelian generalizations of Poincaré type supersymmetries are consistent with the presence of two timelike dimensions [15] compare also [16], [17], [18], [19], [20], [21], [22], [23], [24].

Although 6-dimensional spacetime cannot be applied in the traditional form [25] because one cannot ascertain orthogonality of six dimensions packed together [26], [27], with quantized tuples of dimensions the problem of multiple dimensions of time could be successfully resolved [28], [29].

The fact that the eq. (2) was operationally illegitimate and conceptually incomplete has been conveniently suppressed, and in order to defy logic, mathematics and scientific common sense some unnamed "geniuses" in the past invented fake theorem called path-independence theorem (PIT) [4]. The PIT is just the proverbial tip of the abstract iceberg of blatant mathematical misconceptions.

According to the PDR, operationally correct work done rate should be evaluated [5] as:

$$
\begin{aligned}
& d W=-F \cos 2 \alpha d r+2 F r \sin 2 \alpha d \alpha-r \cos 2 \alpha d F=-d W(r)+d W(\alpha)- \\
& d W(F)
\end{aligned}
$$

where $\alpha$ is the angle of visibility of trajectory/orbit of a satellite (or that of an electron in an atom in the Bohr's classical picture) as seen from local gravity center when sweeping its perihelion radius. The formula (3) contains the usual radial work done rate $\mathrm{dW}(\mathrm{r})$ as well as two formerly disregarded components of total work done, namely the angular nonradial rate $\mathrm{dW}(\alpha)$ and the linear nonradial rate $\mathrm{dW}(\mathrm{F})$ of the total work done. Note that the rates of work done pertain also to rates of the field's potential energy that is spent on the work done by the given radial/center-bound force field.

Notice that the sign of the angular nonradial term is opposite to the signs of the attractive terms - the usual radial term and the linear nonradial term. This fact gives rise to - formerly unknown - angular nonradial repulsive force of the usual radial gravitational field in which it is intrinsically embedded. The repulsive effect of the angular nonradial part is a repulsive twist. Although in this presentation the force field is of gravitational nature, it could be of electrostatic origin too.

Since no law of physics is complete but depends on the depth of inquiry into the phenomena under investigation, I shall specify the particular level/depth of inquiry used in this presentation. At the present depth of inquiry, I will consider only gravitational interactions happening within the usual radial/center-bound force field due to a nonrotating celestial body with fixed mass $\mathrm{M}$ and assumed as constant average density of matter of the source mass $M$ that generates the field.

When substituting magnitude of the usual radial force vector $\mathbf{F}$ of the gravitational force field

$$
\mathrm{F}=|\mathbf{F}|=\frac{-\mathrm{GMm}}{\mathrm{r}^{2}}
$$

into the differential equation (3) and solving it with respect to the radial distance $r$ alone, we obtain

$$
\mathrm{W}(\mathrm{r})=\frac{\mathrm{GMm}}{\mathrm{r}}
$$

which is the usual (i.e. radial-only) amount (hence functional) of the interacting potential energy at the generic distance $r$ to the trajectory/orbit of the mass $m$ (i.e. with $\alpha=0$ ) that is just passing by (or orbiting) the (assumed as big) source mass $\mathrm{M}$ that generates the locally dominant gravitational force field. $\mathrm{G}$ is the usual gravitational constant.

Solving the equation (3) with respect to the originally planar angle $\alpha$ (of visibility of the orbit or trajectory) alone, which during motion of the mass $\mathrm{m}$ evolves into a twisting - when viewed 
from the gravity center associated with the source mass $M-$ spherical/spheroidal angle $\theta$ giving thus the angular distance $\lambda=r \theta$ measured along an equipotential surface, we obtain:

$$
\mathrm{W}(\mathrm{Q}(\lambda))=\frac{-4 \mathrm{kGMmr} \mathrm{p}_{\mathrm{p}}^{2} \lambda}{3 \mathrm{Qr}}=\frac{-\mathrm{kuGMm} \lambda}{\pi \mathrm{r}_{\mathrm{p}} \mathrm{Qr}^{4}}
$$

which is the angular nonradial amount of potential energy spent on the work done by the field along the equipotential surface a the perihelion distance $r_{p}$ that is also codetermined by the radial distance $r$, and the (assumed as average - at the present depth of inquiry into the gravitational phenomena) density of matter $\mathrm{Q}$ of the source mass $\mathrm{M}$ and the perimeter volume $v$ enclosed within the perihelion [6], [3]. The value of proportionality constant $\mathrm{k}$ proved to be 1 for practically spherical celestial bodies such as our Sun and Earth [2]. It is also needed for equalizing the units used (such as radians or angular degrees).

Here $v$ is the perimeter volume enclosed by the fixed distance $r_{p}$ to the perihelion from the local gravity center of the mass $M$. The equipotential distance $\lambda=r \theta$ is codetermined also by the radial distance $r$ (which is limited from below by the radius of perihelion $\mathbf{r}_{\mathrm{p}}$ ) and by the spherical angle $\theta$ (which can be curvilinear, i.e. spheroidal spherical in general) that corresponds to the angular equipotential path $\lambda$. While the angle $\alpha$ (of visibility of the mass $m$ that moves on trajectory or orbit) is anchored at the center of gravity of the locally dominant field generated by the source mass $\mathrm{M}$, the angle $\theta$ is anchored at the trihedron that moves with the mass $\mathrm{m}$. The angle $\alpha$ is being twisted, whereas the angle $\theta$ remains steady within the trihedron, which is being twisted. Hence by anchoring the angle $\theta$ at the trihedron, it has been made fixed therein.

The constant density of matter functional Q, which is practically fixed parameter for planets, is nevertheless also a function of exposure. Although fixed function of exposure does not change its own value, it allows accumulation of the (nonradial) impact it exerts on the small orbiting test mass $\mathrm{m}$ along the angular distance or during the time of its exposure to the influence of the big mass $\mathrm{M}$. It is indeed analogous to exposure to heat or radiation.

Note that exposure to impact of the mass $M$ was unknown in former physics due to the faulty formula (2), even though it was well known fact that large seas/oceans and landmasses do affect propagation of radio signals of the land-based differential positioning system used for navigation of sea-faring ships in the past (see select references in [2] that attest to presence of the latter effect).

It is important to realize that both equations (5) and (6) are functional equations (as opposed to function-based equations), because they are just formulas to be calculated from, even though I have marked them as functions depending on independently varying variables $r$ and $\lambda$, respectively. There was no clear distinction between function-based dependence and functional-based dependence (which I would prefer to call determination) in traditional mathematics, presumably because the distinction between functions and functionals is of situational nature. Integrals are functionals, but compound expressions - such as the eq. (6) - usually contain both functionals and functions. The density of matter $Q$ in the eq. (6) is both a functional and a function of exposure that depends on $\lambda$.

Although functional equations were usually called functions, one can see that the expressions standing on the left-hand side (LHS) are just functionals whose values are to be calculated from evaluation of the expressions standing on the RHS of the equality (or substitution) sign. Hence the conventional embrace of functionals in square brackets is not quite appropriate for this presentation, however, because when we combine the two equations the variable $r$ designating function $W(r)$ in the first one is the same as the variable $r$ that merely determines the function $W(Q(\lambda))$ in the second equation. The conventional notation thus breaks down. The functional status of the two equations stems from the fact that they pertain to accumulated amounts, not to differentials, which of necessity pertain to functions differentiated therein. I have demonstrated operational difference in treatment of functions versus functionals in [8]. 
In order to put the eq. (6) on equal footing with the eq. (5) we should substitute the angle $\theta=\lambda / r$ and disregard the perihelion radius $r_{p}=r$. These substitutions reduce thus the eq. (5) to the following:

$$
\mathrm{W}(\mathrm{Q}(\theta))=-\mathrm{K} \frac{\mathrm{GMm} \theta}{\mathrm{Qr}}
$$

where $\mathrm{K}$ is the consolidated constant coefficient replacing all the other constants in (6).

As one can see the angular nonradial part has opposite signs in both the eq. (3) - on the level of forces- as well as when comparing the eq. (5) with (6)-(7) - on the potential energy level which upholds the mathematical suggestion (or theoretical hint, if you will) that they indeed correspond to opposite, i.e. attractive and repulsive interactions, respectively. We have thus no formal overlapping of effects of these two interactions.

\section{ONCE EQUALIZED MASS IS INTRODUCED, DENSITY OF MATTER EXPLICITLY AFFECTS RADIAL GRAVITATIONAL INTERACTIONS TOO}

Nonoverlapping of radial and nonradial interactions permits equalization of the impact of the essentially inertial source mass $M$ for stars and planets that move at different nonrelativistic local speeds (i.e. with $\mathrm{v}<<\mathrm{c}$, where $\mathrm{c}$ is the speed of light in vacuum) by introducing a certain equalized mass $\mathcal{M}$ which would characterize all celestial bodies irrespective of their relative motion or their material composition.

The equalized mass shall associate the notion of mass with the material substance out of which the bodies are made (or bulk - as Newton has called it, with density of matter) rather than with motion of the massive body M. Since density of matter is defined as ratio of mass of the given body to the equivalent to it mass of water $\mathrm{Q}=\mathrm{M} / \mathrm{M}_{\mathrm{w}}$, let us designate for now the equalized mass as equal to that of water $\mathcal{M}=\mathrm{M}_{\mathrm{w}}$ under the same physical circumstances. This way masses of all planets within our solar system could be quite meaningfully compared [3].

To be honest, I am not doing it for a mere convenience. The traditional comparisons of masses of planets, each of which orbits our sun at different speeds, resulted in rather infantile inferences, some perpetuated for centuries [3]. Only if celestial bodies are considered as if they were made of the same material substance, we could avoid unnecessary bias and make meaningful inferences from comparisons of these bodies.

The equalized mass could thus truly be the gravitational mass. The former physics was obsessed with proving that inertial and gravitational mass were the exactly same. That was exercise in futility. Only when the traditional notion of inertial (i.e. motion-affected) mass is separated from the notion of material substance (that is usually determined by density of matter, at least in macrophysics), we can split the concept of mass into two quite distinct magnitudes: equalized mass and density and density of matter. Both of these measures of what Newton called 'quantity of matter', have influence on gravitational interactions, but each impacts gravitation differently. Now mass and matter have precise but distinctively different meanings, even though they jointly - as Newton stipulated - characterize celestial bodies. The equalized mass delivers the radial pull whereas the density of matter supplies the angular twist. Now we can handle these notions mathematically, instead of babbling about the former, imprecisely defined inertial and gravitational mass philosophically.

With this in mind, we can rewrite the two equations (5) and (7), respectively, as follows:

$$
\begin{aligned}
& \mathrm{W}(\mathrm{r})=\frac{\mathrm{G} N \mathrm{mQ}}{\mathrm{r}} \\
& \mathrm{W}(\theta)=-\mathrm{K} \frac{\mathrm{G} N \mathrm{~m} \theta}{\mathrm{r}}
\end{aligned}
$$


which vindicates that ingenious Newton's assumption that density of matter should really matter for all gravitational interactions, as it should. Notice that with the equalized mass $\mathcal{M}$ in place, the usual Newtonian radial gravitational potential energy explicitly depends on the constant/average density of matter Q, whereas the angular nonradial potential energy in eq. (9) depends on it too, but the dependence is implicit.

Without exaggeration, one might say thus that this introduction of the concept of equalized mass marks the final end of natural philosophy and starts mathematically responsible gravitational physics. Since Einstein has deliberately omitted consideration of nonradial effects - as did all pre$2000 \mathrm{AD}$ theories of gravitation due to blind adherence to the faulty former definition of potential energy - even his theory was incomplete. Since all those theories virtually defied the PDR, they were mathematically irresponsible, even if their authors were unaware of that.

\section{EQUALIZED MASS MARKS THE END OF NATURAL PHILOSOPHY}

Newton has postponed publication of his Principia by some 20 years, presumably because he could not resolve the dependency depicted in the above eq. (8). I am not blaming Newton for "shady" work. Quite to the contrary. His was clearly superb mathematical genius, but it required work of many others, both mathematicians and physicists, to get to the alternative formulation portrayed in the eq. (8).

One can see that depending on the depth of inquiry into gravitational phenomena, the very same formula can be expressed as in the eq. (5) or (8). Formulation of laws of mathematics and of physics surely depends on one's depth of inquiry. Moreover, those different formulations may not always be entirely equivalent to each other. The fact that the different formulations may have some discrepant (i.e. not quite equivalent conceptual consequences) was yet another reason for pursuing extensions of well-established laws of physics via uncovering some previously hidden variables [8].

For us today the lesson is this: Do not try to "analyze" mathematical issues (or perhaps make inferences from mathematical assertions) by using linguistic tools alone (i.e. by deciding the inner meanings of mathematical concepts and inferences by using dictionary of any language alone), because it is the synthetic matching of structures and corresponding to them operational procedures that permits drawing valid conceptual inferences. To draw conclusion that radial force fields cannot have any nonradial interactions may sound good from a purely linguistic standpoint, yet mathematically it is not only possible but in fact, it is unavoidable in general [9].

The most fundamental thesis of mathematical intuitionism in almost all its variants says that existence in mathematics coincides with constructability [30]. Although buildup of geometry free from logical contradictions is possible [31], which makes it internally consistent [32], these conditions can ensure only inner noncontradictions. To validate its noncontradictory coexistence with other theories requires more than that, if the theory is to be realistic [33]. Constructivism started from the (mistaken, if you ask me) belief that mathematics is simply invented rather than discovered [34]. Constructivist approach to mathematics views theorems as mere reports on intuitive constructions [35]. There is nothing in intuition that could prevent making misguided or even nonsensical constructions, however. The fake path-independence theorem was never proved but only outlined with appeal to intuition - yet it was perpetuated in mathematics and physics for some two centuries (if counting from 1773 AD) [4].

The new synthetic mathematics adds to the requirement of constructability also the extra necessity that structures match operational procedures performed on the structures. The reason for the addition is to avoid existtential postulates, because it is not unimaginagble to construct a - hard to identify - nonsense. For existential postulates tacitly create an artificial reality, which may or may not actually exist, even though it could be formally constructed. Constructability is surely necessary, but it is not suffiecient condition to ascertain the validity of the construct. If a structure is formally constructed but cannot be operated on then this likely means that the theory that supplied theb tools for the construction may not be valid. The pertinent examples of arbitrary higher- 
dimensional constructs, which can defy some proven achievements of Abel, Galois and Lagrange, are given in [28], [29].

Nobody has patent on "correct" ways of thinking. It is also worth remembering that we learn mathematics at the - essentially analytical - level of comprehension. To understand it and its various conceptual consequences, truly synthetic approach is necessary (which used to be depicted among higher synthetic skills on then "new" Bloom's taxonomy pyramid when I was studying it).

\section{CAN CONSEQUENCES OF EQUALIZING MASS BE OBSERVED?}

When mass is equalized, we can meaningfully combine the usual radial gravitational formula with the angular nonradial functional by superposing their formally independent effects:

$$
\mathrm{W}(\mathrm{r}, \theta):=\mathrm{W}(\mathrm{r})+\mathrm{W}(\theta)=\frac{\mathrm{G} N \mathrm{~m}}{\mathrm{r}}(\mathrm{Q}-\mathrm{K} \theta)
$$

which can be further generalized via the equalized radial potential energy term U(r) as:

$$
\mathrm{W}(\mathrm{U}(\mathrm{r}), \theta)=\mathrm{U}(\mathrm{r})(\mathrm{Q}-\mathrm{K} \theta) \text { with } \mathrm{U}(\mathrm{r})=\frac{\mathrm{G} N \mathrm{~m}}{\mathrm{r}}
$$

to show its curious dependence of gravitational interactions on the trihedron-based angular nonradial twisting angle $\theta$.

The equations (10) and (11) are only preliminary not final, for the angular nonradial potential energy has been derived for rotating satellites but the mass source of the locally dominant fields was assumed as nonrotating. At a deeper than the present level of inquiry into phenomena of potential energy interchange, more comprehensive equations shall be presented. This shall be done elsewhere, for it requires some preparatory steps along the line of thought already outlined in [36] and [37].

The eq. (11) could be called pull-and-twist functional equation of orbital gravitational interactions. It shall be further developed elsewhere.

\section{CONJECTURES FROM PULL-AND-TWIST EQUATION OF GRAVITATION}

The eq. (11) with equalized mass suggests the possibility that density of matter of the mass source of the locally dominant gravitational force field could be counterbalanced by orbiting masses. This could happen only when the locally dominant mass source is very light, such as the gas giant Saturn and perhaps Jupiter, which has large gas coating.

This conjecture is suggested by the fact that the angular nonradial part, whose overall effect is dissipative (when spoken of it in terms of energy exchange), or twistingly repulsive when it is considered in terms of forces, could - according to the eq. (11) - stabilize orbiting debris, provided the density of matter of the locally dominant planet can be induced to vary by the angular nonradial twist exerted on the debris. The factor $(\mathrm{Q}-\mathrm{K} \theta)$ in the eq. (11) makes the possibility plausible indeed, at least for gaseous and significantly gas-coated planets.

Without going into further details, which besides some input from astronomy would require also experimental evidence pertaining to rings surrounding the two planets, I would conjecture that their rings are maintained because the gas surrounding those planets could be easily condensed as well as decompressed due to potential energy exchange between those planets and their moons and outer rings. 
Perhaps an indication of that could (theoretically) be achieved by measuring nonradial frequency decrease in rays coming from distant stars when they are grazing the surfaces (or designated boundaries) of those gas-coated planets. However, since past experiments did not take into account density of matter, their reliability may not be ascertained.

Although delineating boundaries of gas-coated giants may be difficult, the possibility of such an energy counterbalancing act might also be discovered by measuring the (presumably varying) emissions of electromagnetic radiation during such forced variations of potential energy distributions. The present author is aware of emissions of electromagnetic radiation (at least from Jupiter) but has no access to any relevant data and is not familiar with details of emissions of the radiation, however.

By the same token, I would conjecture that the exchange of interaction energy contributes to twisting of arms of spiral galaxies, although I cannot provide evidence for that.

\section{CONCLUSIONS}

When equalized mass is introduced into the classic Newtonian equation of essentially radial gravitation, density of matter emerges in it by default. In the sense, the assumed as constant/average density of matter was as if formerly hidden variable. It is stipulated that relying on equalized mass could have certain distinctively predicted consequences, which might be observed, at least in principle.

\section{References}

[1] Newton I. Principia. Edited and commented by Stephen Hawking. Philadelphia: Running Press, 2002, pp.1,321.

[2] Czajko J. On Conjugate Complex Time II: Equipotential Effect of Gravity Retrodicts Differential and Predicts Apparent Anomalous Rotation of the Sun. Chaos, Solitons \& Fractals 11 (2000) 2001-2016.

[3] Czajko J. Galilei was wrong: Angular nonradial effects of radial gravity depend on density of matter. International Letters of Chemistry, Physics and Astronomy 30 (2014) 89-105 http://www.scipress.com/ILCPA.30.89.pdf.

[4] Czajko J. Path-independence of work done theorem is invalid in center-bound force fields. $\begin{array}{lllll}\text { Studies in } & \text { Mathematical } & \text { Sciences } & \text { 7(2) }\end{array}$ http:/cscanada.net/index.php/sms/article/view/j.sms.1923845220130702.2469/5279.

[5] Czajko J. Equipotential energy exchange depends on density of matter. Studies in Mathematical $\begin{array}{llll}\text { Sciences } & 7(2) & \text { (2013) }\end{array}$ http://cscanada.net/index.php/sms/article/view/j.sms.1923845220130702.2752/5336 .

[6] Czajko J. Operationally complete work done suggests presence of potentials corresponding to repulsive forces. International Letters of Chemistry, Physics and Astronomy 37 (2014) 16-30 http://www.scipress.com/ILCPA.37.16.pdf.

[7] Czajko J. On the Intrinsic Gravitational Repulsion. Chaos, Solitons \& Fractals 19 (2004) 683700.

[8] Czajko J. Mathematical gateway to complementary hidden variables in macrophysics. International Letters of Chemistry, Physics and Astronomy 50 (2015) 117-142 http://www.scipress.com/ILCPA.50.117.pdf.

[9] Czajko J. Radial and nonradial effects of radial fields in Frenet frame. Applied Physics Research 3(1) (2011) 2-7 http://www.ccsenet.org/journal/index.php/apr/article/viewFile/9555/7421 
[10] Birkhoff G. (Ed.) (1973) A source book in classical analysis. Cambridge, MA: Harvard Univ. Press, pp.335, 360.

[11] Kar K.C. Notes on the theory of relativity. Ind. J. Theor. Phys. 17(1) (1969) 1-11.

[12] Kar K.C. \& Dutta C. On the theory of relativity. Ind. J. Theor. Phys. 16(1) (1968) 1-6.

[13] Taylor J.G. Do electroweak interactions imply six extra time dimensions? J. Phys. A: Math. Gen. 13 (1980) 1861-1866.

[14] Ivashchuk V.D. \& Melnikov V.N. Multitemporal Generalization of the Schwarzschild Solution. Int. J. Mod. Phys. D4 (1995) 167-173.

[15] Bars I., Deliduman C. \& Minic D. Supersymmetric two-time physics. Phys. Rev. D 59 (1999) 125004-9.

[16] Ord G.N. Fractal space-time: a geometric analogue of relativistic quantum mechanics. J. Phys. Math. Gen. 16 (1983) 1869-1884.

[17] Gubarev, V.V. Multidimensional time and spectral concors of stationary processes. Nonparametric estimates. Russ. Phys. J. 38(9) (1995) 890-893.

[18] Mirman, R. Comments on the dimensionality of time. Found. Phys. 3(3) (1973) 321-333.

[19] Bars I. Standard model of particles and forces in the framework of two-time physics. Phys. Rev. D 74 (2006) 085019.

[20] Bars I. Twistor superstring in two-time physics. Phys. Rev. D 70 (2004) 104022.

[21] Bars I. \& Deliduman C. High speed gauge fields and two-time physics. Phys. Rev. D 64 (2001) 045003.

[22] Bars I. Two-time physics in field theory. Phys. Rev. D 62 (2000) 046007.

[23] Bars I., Deliduman C. \& Andreev O. Gauged duality, conformal symmetry, and spacetime with two times. Phys. Rev. D 58 (1998) 066004.

[24] Bars I. \& Kounnas C. String and particle with two times. Phys. Rev. D 56 (1997) 3664-3671.

[25] Weinberg N.N. On some generalizations of the Lorentz transformation. Phys. Lett. 80A(2-3) (1980) 102-104.

[26] da Costa R.C.T. Constraints in quantum mechanics. Eur. J. Phys. 7 (1986) 269-723.

[27] da Costa R.C.T. Constraints in quantum mechanics. Phys. Rev. A 25 (1982) 2893-2900.

[28] Czajko J. Operational constraints on dimension of space imply both spacetime and timespace. International Letters of Chemistry, Physics and Astronomy 36 (2014) 220-235 http://www.scipress.com/ILCPA.36.220.pdf.

[29] Czajko J. Operational restrictions on morphing of quasi-geometric 4D physical spaces. International Letters of Chemistry, Physics and Astronomy 41 (2015) 45-72 http://www.scipress.com/ILCPA.41.45.pdf.

[30] Fraenkel A.A., Bar-Hillel Y. \& Levy A. Foundations of set theory. Amsterdam: NorthHolland, 1973, p.220.

[31] Pasch M. Grundfragen der Geometrie. J. reine angew. Math. 147 (1917) 184-190.

[32] Heyting A. Axiomatic projective geometry. Groningen: Noordhoff, 1980, p5.

[33] Wang H. Reflections on Kurt Gödel. Cambridge, MA: The MIT Press, 1987, p.296. 
[34] Calder A. Constructive mathematics. Sci. Am. 241 (1979) 146.

[35] Körner S. The philosophy of mathematics. London: Hutchinson Univ. Library, 1960, p.135.

[36] Czajko J. Angular nonradial vs. usual radial potential energy quotient. International Letters of $\begin{array}{llllll}\text { Chemistry, } & \text { Physics } & \text { and Astronomy } & 52 & \text { (2015) }\end{array}$ http://www.scipress.com/ILCPA.52.172.pdf.

[37] Czajko J. Linear magnitudes estimated via energy were likely overestimated by over 3.48\%. International Letters of Chemistry, Physics and Astronomy 32 (2014) 32-41 http://www.scipress.com/ILCPA.32.32.pdf . 